\title{
Noninvasive in vivo optical coherence tomography tracking of chronic otitis media in pediatric subjects after surgical intervention
}

Guillermo L. Monroy

Paritosh Pande

Ryan M. Nolan

Ryan L. Shelton

Ryan G. Porter

Michael A. Novak

Darold R. Spillman, Jr.

Eric J. Chaney

Daniel T. McCormick

Stephen A. Boppart 


\title{
Noninvasive in vivo optical coherence tomography tracking of chronic otitis media in pediatric subjects after surgical intervention
}

\author{
Guillermo L. Monroy, ${ }^{\text {a,b }}$ Paritosh Pande, ${ }^{b}$ Ryan M. Nolan, ${ }^{b}$ Ryan L. Shelton,, b,c Ryan G. Porter, ${ }^{\text {d,e }}$ \\ Michael A. Novak, ${ }^{d, e}$ Darold R. Spillman Jr., ${ }^{b}$ Eric J. Chaney,, Daniel T. McCormick, ${ }^{f}$ and \\ Stephen A. Boppart ${ }^{a, b, c, e, *}$ \\ aUniversity of Illinois at Urbana-Champaign, Department of Bioengineering, Urbana, Illinois, United States \\ ${ }^{\mathrm{b} B e c k m a n}$ Institute for Advanced Science and Technology, Urbana, Illinois, United States \\ 'University of Illinois at Urbana-Champaign, Department of Electrical and Computer Engineering, Urbana, Illinois, United States \\ ${ }^{\mathrm{d} C}$ Carle Foundation Hospital, Department of Otolaryngology, Urbana, Illinois, United States \\ eUniversity of Illinois at Urbana-Champaign, Carle-Illinois College of Medicine, Urbana, Illinois, United States \\ ${ }^{\mathrm{f} A d v a n c e d M E M S, ~ S a n ~ F r a n c i s c o, ~ C a l i f o r n i a, ~ U n i t e d ~ S t a t e s ~}$
}

\begin{abstract}
In an institutional review board-approved study, 25 pediatric subjects diagnosed with chronic or recurrent otitis media were observed over a period of six months with optical coherence tomography (OCT). Subjects were followed throughout their treatment at the initial patient evaluation and preoperative consultation, surgery (intraoperative imaging), and postoperative follow-up, followed by an additional six months of records-based observation. At each time point, the tympanic membrane (at the light reflex region) and directly adjacent middle-ear cavity were observed in vivo with a handheld OCT probe and portable system. Imaging results were compared with clinical outcomes to correlate the clearance of symptoms in relation to changes in the image-based features of infection. OCT images of most all participants showed the presence of additional infection-related biofilm structures during their initial consultation visit and similarly for subjects imaged intraoperatively before myringotomy. Subjects with successful treatment (no recurrence of infectious symptoms) had no additional structures visible in OCT images during the postoperative visit. OCT image findings suggest surgical intervention consisting of myringotomy and tympanostomy tube placement provides a means to clear the middle ear of infection-related components, including middle-ear fluid and biofilms. Furthermore, OCT was demonstrated as a rapid diagnostic tool to prospectively monitor patients in both outpatient and surgical settings. @ 2017 Society of Photo-Optical Instrumentation Engineers (SPIE) [DOI: 10.1117/1.JBO.22.12.121614]
\end{abstract}

Keywords: otitis media; prospective observation; optical coherence tomography; surgical intervention; tympanostomy; myringotomy; biofilm.

Paper 170327SSRR received May 21, 2017; accepted for publication Dec. 15, 2017; published online Dec. $23,2017$.

\section{Introduction}

Middle-ear infections, commonly known as otitis media (OM), affect more than $80 \%$ of children. ${ }^{1}$ Current studies estimate the diagnostic accuracy for $\mathrm{OM}$ by an expert physician using standard otoscopy at $60 \%$ to $70 \% .^{2}$ Existing technology used to diagnose $\mathrm{OM}$ is limited primarily to illumination and magnification devices, ${ }^{3}$ such as otoscopes or surgical microscopes, which only provide a surface view of tissue. Diagnosis using these tools relies on accurately interpreting visual features of the tympanic membrane (TM) or the accurate detection of the presence of any middle-ear effusion (MEE). Pneumatic otoscopy, while considered the gold standard for OM diagnosis, is difficult to perform correctly and is often not utilized in daily clinical practice. ${ }^{4}$ Other acoustic techniques, such as tympanometry and acoustic reflectometry, provide additional insight into the functional behavior of the TM and middle-ear mobility. While tympanometry can help to infer middle-ear dysfunction and provide slightly improved diagnostic accuracy over otoscopy alone, tympanometry results are still recommended to be considered alongside otoscopy findings. ${ }^{5}$ Persistent or recurrent cases of

*Address all correspondence to: Stephen A. Boppart, E-mail: boppart@illinois .edu
$\mathrm{OM}$ are treated with tympanostomy tube (TT) placement, one of the most commonly performed surgeries in children requiring general anesthesia. ${ }^{6,7}$ While $\sim 6 \%$ of all children in the US will receive TT before the age of 2 , there are reports that physicians often inconsistently recommend TT placement when verified against treatment guidelines, ${ }^{8}$ although varying subpopulations and differing treatment implementation strategies across the US explain this variance. ${ }^{8-12}$

This study is motivated by previous efforts that used optical coherence tomography (OCT), a noninvasive and highresolution medical imaging technology, to visualize the TM and middle ear. Past studies have used OCT in surgical settings, ${ }^{13-15}$ although its use has previously been limited mainly to ex vivo ${ }^{16-19}$ tissue due to technical challenges and physical limitations of light delivery to the ear. Our group has addressed many of these challenges and performed both animal ${ }^{7,20}$ and human ${ }^{6,21-25}$ studies. Recently, one study ${ }^{21}$ identified and visualized additional infection-related structures affixed to the TM in nearly every case of chronic OM, which led to our current interest to understand longitudinal changes in the observed structures following surgical intervention. 
The pathogenesis of these additional infection-related structures observed in OCT images is unclear. Yet, the presence of biofilms during infection in the respiratory tract and upper airway, including the adenoids and middle-ear cavity (MEC), is understood and well established..$^{20,26-34}$ There remains, however, a lack of understanding of the specific role that biofilms play during "healthy" or "infected" conditions in the middle ear, as previously it has not been possible to regularly and longitudinally observe these biofilms in vivo. Biofilms are communities of bacteria enclosed in a self-produced extracellular polymeric substance. ${ }^{35}$ Biofilms protect bacteria from host immune response mechanisms ${ }^{36-43}$ and antibiotic treatments. Given the possible virulence, persistence, and/or recurrence of biofilmassociated infection in the ear, understanding the mechanisms that drive the infection process was recently declared a grand challenge in pediatric otolaryngology. ${ }^{44}$

In this study, an OCT system combined with video-based surface imaging of the TM was used to prospectively observe patients through consecutive preoperative, surgical, and postoperative visits to quantitatively characterize and assess the efficacy of surgical TT placement to clear middle-ear infection. It is expected that a patient with a successful surgical intervention would have no further clinical symptoms, and OCT images would be clear of any image-based features that would be associated with $\mathrm{OM}$, including any MEE or biofilm. In the presence of persistent or recurrent clinical symptoms of OM, we expect to find OCT image evidence of persistent or recurrent MEE and/or middle-ear biofilm.

\section{Materials and Methods}

\subsection{Subject Population and Recruitment}

Subjects were recruited from the outpatient pediatric population of Urbana-Champaign, Illinois, visiting Carle Foundation Hospital for treatment in the Department of Otolaryngology. Twenty-five ${ }^{25}$ subjects were prospectively observed at both secondary care and outpatient surgical centers for $\sim 6$ months, with 6 months of additional medical record-based follow-up. Subjects were imaged with OCT at up to three possible time points in their treatment: (1) patient evaluation and preoperative surgical consultation, (2) surgery (intraoperative imaging), and (3) postoperative follow-up.

All participants provided informed consent and assent in accordance with protocols approved by the institutional review boards of Carle Foundation Hospital and the University of Illinois at Urbana-Champaign. The standard-of-care treatment provided was according to recommended national treatment guidelines for acute $\mathrm{OM},{ }^{45} \mathrm{OM}$ with effusion (OME), ${ }^{46}$ and TT placement. ${ }^{47}$ Subjects diagnosed with chronic or recurrent $\mathrm{OM}$ and participating in this study had, at a minimum, multiple infections within a 3- to 6-month time period and/or a persistent MEE for 3 months, as well as concerns about hearing, speech, and/or cognitive development. All subjects were $<18$ years of age and were excluded if their temperament or discomfort prohibited imaging with the handheld OCT probe. No exclusions were made based on gender or race.

\subsection{Handheld Probe and Portable OCT System}

A custom-built handheld probe and portable OCT system (Fig. 1) were used to image subjects. The system utilized a broadband optical source (Superlum, Ireland) centered at
$860 \mathrm{~nm}$ with an approximate bandwidth of $135 \mathrm{~nm}$ at full width at half maximum, which provided an axial resolution of $\sim 2.4 \mu \mathrm{m}$ and a transverse resolution of $15 \mu \mathrm{m}$, in air. A line scan camera-based spectrometer (Wasatch Photonics) provided data to process cross-sectional B-mode images. The OCT system emitted an optical power of $2.5 \mathrm{~mW}$ onto the TM, which is well below ANSI standard safety limits. ${ }^{48}$ A separate, commercial video otoscope (Welch Allyn) was used to document surface images of the TM. Additional details regarding the system can be found in a prior publication. ${ }^{49}$

\subsection{Image Collection Protocol}

The imaging protocol aimed to minimize any interruption to the standard-of-care examination procedures at each imaging site and ensure that pediatric subjects would remain as calm and attentive as possible to minimize artifacts in the collected data. At each imaging time point, the handheld probe was inserted into the ear canal and positioned to bring the TM into the field of view. The light reflex region of the TM was then identified and observed with OCT, using the malleus and umbo as anatomical landmarks for positioning. The OCT system utilized a buffer to constantly process and display images for real-time viewing, with a trigger on the handheld probe to save images. Depressing the trigger stored the 40 most recently visualized OCT images for later analysis. Multiple stacks of B-scans were typically acquired at each time point, which later allowed a representative image to be selected for analysis. In place of saving all visualized data or needing to manipulate on-screen buttons to initiate saving, the use of a constantly updating buffer and trigger method allowed for more efficient collection of high-quality images. Primarily, this ensured that collected OCT images were taken from the light reflex region of the TM and avoided saving empty or unrelated images during positioning of the handheld probe in the ear canal and around any earwax that may have been present. This also allowed the operator to more successfully manage patient movement that occurs throughout the examination and limit the acquisition of images with artifacts when possible. OCT images were not interpreted or analyzed
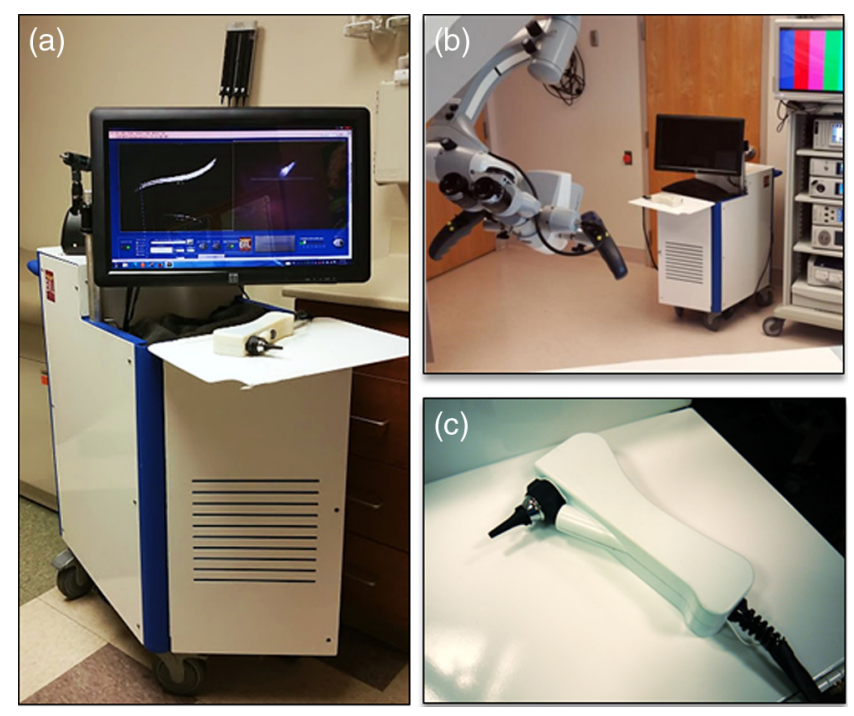

Fig. 1 Portable optical imaging system and handheld OCT probe (a) within the specialist's clinic and (b) within the operating theater. (c) Handheld probe utilizes standard interchangeable ear specula tips that are replaced between patients. 
for content before or during collection. Physicians did not utilize OCT images for clinical decision-making.

In the otolaryngology clinic, imaging was performed after regularly scheduled examinations, such that the physician could first formulate an unbiased treatment plan. During surgery, OCT imaging was performed intraoperatively after clearing of any cerumen, part of the standard-of-care procedure in this setting, and prior to myringotomy or TT placement. Otoscopic examination of the TM requires an unobscured line of sight through the ear canal, although a view that is not entirely clear of cerumen (ear wax) or small hairs is common. However, additional cerumen removal specifically to improve OCT imaging in the otolaryngology clinic was not performed. Standardof-care practices in the clinic dictate that cerumen is removed only if there is a severe obstruction of the physicians' view of the TM for otoscopy. ${ }^{50}$ Furthermore, cerumen removal incurs additional costs for the patient, estimated in 2012 to be $\sim \$ 35$ USD. ${ }^{51}$ In either clinic or surgical setting, imaging both ears took $\sim 5 \mathrm{~min}$.

\subsection{Image Processing and Analysis Protocol}

OCT image processing and analysis were performed by experienced readers familiar with OCT and OCT ear imaging, although there was no training specific to this study. Readers were provided with sequentially numbered OCT image stack files and digital otoscopy images from a single time point for analysis and were blinded to the imaging time point and clinical diagnoses during analysis. Images were removed from consideration and not analyzed if they contained artifacts (saturations, reflections, or wrapping), were acquired from the ear canal, positions on the TM other than the light reflex, or were out of focus. From the remaining set of images, a representative image that best demonstrated the overall findings from the time point was selected by the reader to maximize image quality and clarity for display and analysis.

Representative images were analyzed to determine the presence or absence of a biofilm or MEE at each time point for each subject using methods determined in a previous publication. ${ }^{21}$ Quantitative features in OCT images that demonstrated the morphological changes of chronic OM include the presence of additional structures within the MEC, such as overall thickening due to the presence of biofilm or MEE, presenting as dense layered structures adhered to the TM, or point-like scatterers within the MEC, respectively. Clearly, defining the interior mucosal surface of the TM and interface between these additional structures can be difficult in some cases if refractive index matching occurs between the TM and middle-ear fluid or biofilm. Similarly, it may not be possible to visualize deeper into the MEC due to the highly scattering nature of more purulent middle-ear fluid. Structures external to the MEC and within the ear canal, such as earwax, were not considered and were mostly unrelated to $\mathrm{OM}$ infection status.

After analysis, images were sorted into a secure repository and organized by subject number, date, and time point. Reports containing clinical diagnosis and findings were collected, organized, and analyzed separately from OCT data at a later time. For the purposes of this study, OCT imaging results were then analyzed in conjunction with findings from patient medical and/or surgical reports to correlate the presence or absence of a middleear biofilm with clinical symptoms of OM and patient outcomes.

\section{Results}

Twenty-five subjects (Table 1) were imaged in this study, which concluded without any adverse events. Patient history was available for any performed examination or procedure. Risk factors indicate an increased likelihood of recurrent OM. Subjects were split into two groups: the "C" (complete) group, and "P" (partial) group. Six subjects in the $\mathrm{C}$ group ( $\mathrm{C} 1$ to $\mathrm{C} 6$ ) were observed at all three time points: preoperative consultation, intraoperatively, and at the follow-up exam. Nineteen subjects in the P group (P1 to P19) were observed at the preoperative consultation visit and then either did not receive surgery (5/19), did not return for follow-up exam after surgery $(5 / 19)$, or were unable to be imaged given scheduling conflicts $(9 / 19)$, which is clarified in the "observed visits" column. The recurrence column indicates treatment outcome through any OM-related visit after surgery to the primary care or otolaryngology clinic, which are further described in the footnote of Table 1.

A simplified visual breakdown of subjects enrolled in this study is presented in Fig. 2, organized by the day of observation. All subjects presented initially with a history of recurrent OM or Eustachian tube dysfunction (ETD) and were observed throughout their treatment with OCT. On their day of preoperative consultation, 92\% (23/25) of subjects had a biofilm identified with OCT. On the day of surgery, 11 subjects were observed with OCT, and $91 \%(10 / 11)$ of subjects had a biofilm identified with OCT immediately prior to surgical intervention. At the follow-up examination, six subjects returned for OCT imaging. These subjects were compliant with the study schedule and comprised the $\mathrm{C}$ (complete) group, with OCT data and clinical reports available from each time point. Of the subjects in the $\mathrm{C}$ group, two of six subjects had additional structures identified with OCT (subjects C4 and C5), and subject C5 reported recurrence issues of OM. In $67 \%$ (4/6) of subjects, surgery was effective at clearing symptoms of OM and had no biofilm identified with OCT. For the remaining 19 subjects that were noncompliant with the study schedule, only partial data was able to be collected. The P (partial) group included clinical reports and OCT data from the day of consultation and reports if/when care was received at a later unscheduled visit. While 8 of 19 subjects returned for all care, $88 \%$ (7/8) of subjects later reported persistent $\mathrm{OM}$ symptoms and had biofilm-like structures previously identified with OCT at an earlier visit. Six of 19 subjects did not return for follow-up care after receiving TT surgery during the study observation period. While follow-up visits and care are recommended as standard practice and included in the cost of surgery, one review found that only $25 \%$ of patients return for follow-up care, despite guidelines that recommend patients regularly follow-up with otolaryngologists until TT has extruded. ${ }^{52}$ While dependent on the type of TT used, most TTs safely extrude on their own within a year, ${ }^{53}$ though follow-up care may still be needed in the future if complications occur. Five of 19 subjects did not receive TT surgery or have a follow-up visit scheduled.

In the $\mathrm{C}$ group, five out of six subjects (83\%) had no recurrence of symptoms after TT surgery. In four out of six subjects, cross-sectional OCT images at the postoperative follow-up visit showed that the MEC was free of biofilm and MEE, although a biofilm had been visualized with OCT during the preoperative visit and intraoperatively during TT surgery. Figure 3 highlights a representative case where subject $\mathrm{C} 3$ presented with chronic OME and ETD and was prospectively imaged with OCT to identify changes following TT surgery. Figure 3(a) shows 
Table 1 Summary of imaging subjects and clinical history.

\begin{tabular}{|c|c|c|c|c|c|c|c|}
\hline \multirow[b]{2}{*}{ Subject } & \multirow[b]{2}{*}{ Patient history } & \multirow[b]{2}{*}{ Risk factors $^{a}$} & \multicolumn{3}{|c|}{ Observed visits } & \multirow{2}{*}{$\begin{array}{l}\text { Tubes } \\
\text { placed }\end{array}$} & \multirow[b]{2}{*}{ Recurrence } \\
\hline & & & 1 & 2 & 3 & & \\
\hline \multicolumn{8}{|c|}{ Complete datasets } \\
\hline $\mathrm{C} 1$ & Recurrent $\mathrm{AOM}$ and $\mathrm{OME}$, repeated $\mathrm{ABx}$ therapy & 1,3 & $\checkmark$ & $\checkmark$ & $\checkmark$ & $\mathrm{Y}$ & 2 \\
\hline $\mathrm{C} 2$ & ETD and chronic OME, hearing loss & $1,3,4$ & $\checkmark$ & $\checkmark$ & $\sqrt{ }$ & $\mathrm{Y}$ & 2 \\
\hline C3 & ETD and chronic OME, hearing loss & $1,3,4$ & $\checkmark$ & $\checkmark$ & $\checkmark$ & Y & 2 \\
\hline $\mathrm{C} 4$ & Recurrent AOM, ETD & 1,3 & $\checkmark$ & $\checkmark$ & $\checkmark$ & Y & 2 \\
\hline C5 & Recurrent AOM, ETD, hearing loss & 1,3 & $\checkmark$ & $\checkmark$ & $\checkmark$ & $\mathrm{Y}$ & 3 \\
\hline C6 & ETD and recurrent $A O M$, repeated $A B x$ therapy & 1 & $\checkmark$ & $\checkmark$ & $\checkmark$ & $\mathrm{Y}$ & 2 \\
\hline \multicolumn{8}{|c|}{ Partial datasets } \\
\hline P1 & Recurrent $\mathrm{AOM}$ & 1,3 & $\checkmark$ & - & - & Y & 2 \\
\hline P2 & Persistent $\mathrm{OME}$, recurrent $\mathrm{AOM}$ & $1,3,4$ & $\checkmark$ & $\times$ & $\times$ & $\mathrm{N}$ & 2 \\
\hline P3 & Hearing loss, recurrent $\mathrm{AOM}$ & $1,3,4$ & $\checkmark$ & $\checkmark$ & $\times$ & Y & 2 \\
\hline P4 & Speech concern, persistent effusion, recurrent $A O M$ & 1,3 & $\checkmark$ & - & - & $\mathrm{Y}$ & 2 \\
\hline P5 & Speech concern, recurrent AOM, ETD & 1 & $\checkmark$ & - & - & Y & 2 \\
\hline P6 & Hearing loss, hypertrophied adenoid, persistent OME & 1,3 & $\checkmark$ & $\checkmark$ & $\times$ & $\mathrm{Y}$ & 2 \\
\hline P7 & Persistent OME and AOM, ETD & 1,4 & $\checkmark$ & - & - & $\mathrm{Y}$ & 2 \\
\hline P8 & Speech concern, persistent OME & 1 & $\checkmark$ & $\checkmark$ & $\times$ & $\mathrm{Y}$ & 2 \\
\hline P9 & Hearing concerns, AOM after TT placement & $1,2,3,4$ & $\checkmark$ & $\times$ & $\times$ & $\mathrm{N}$ & 3 \\
\hline P10 & AOM after TT placement & $1,2,4$ & $\checkmark$ & $\times$ & $\times$ & $\mathrm{N}$ & 3 \\
\hline P11 & Recurrent OME, AOM after TT placement & $1,3,4$ & $\checkmark$ & $\checkmark$ & $\times$ & $\mathrm{Y}$ & 3 \\
\hline $\mathrm{P} 12$ & Speech concern, recurrent OME & 1,3 & $\checkmark$ & $\times$ & $\times$ & $\mathrm{N}$ & 3 \\
\hline P13 & Recurrent OME, AOM, ETD & 1 & $\checkmark$ & - & $\times$ & $\mathrm{Y}$ & 2 \\
\hline $\mathrm{P} 14$ & Persistent OME, recurrent AOM after TT placement, ETD & $1,2,3$ & $\checkmark$ & $\checkmark$ & $\times$ & $\mathrm{Y}$ & 1,3 \\
\hline P15 & ETD, recurrent OME after TT placement & $1,2,3$ & $\checkmark$ & - & - & $\mathrm{Y}$ & 1,3 \\
\hline P16 & Recurrent OME, ETD & 3,4 & $\checkmark$ & $\times$ & $\times$ & $\mathrm{N}$ & 2 \\
\hline P17 & ETD, recurrent OME, third TT placement & 1,2 & $\checkmark$ & - & - & $\mathrm{Y}$ & 1,3 \\
\hline P18 & Recurrent AOM, persistent OME, ETD & 1 & $\checkmark$ & - & - & $\mathrm{Y}$ & 2 \\
\hline P19 & Speech concern, recurrent $\mathrm{AOM}$ and $\mathrm{OME}$ & 1 & $\checkmark$ & - & - & Y & 2 \\
\hline
\end{tabular}

Note: Observed visits $(1,2,3) ; \checkmark$, attended and scanned participant; $\times$, participant did not attend; -, participant received treatment but OCT imaging was not performed; ABx, antibiotic; AOM, acute otitis media; ETD, Eustachian tube dysfunction; OME, otitis media with effusion; TT, tympanostomy tube; and URS, upper respiratory system.

${ }^{a}$ Risk factors (if reported)-1: Recurrent or persistent issues with OM or ETD, 2: has had TTs in the past, 3: suspect tympanogram or hearing test (hearing loss), and 4: family history or URS issues with asthma/allergies.

${ }^{\mathrm{b}}$ Recurrence factors (if reported)-1: Multiple placements of TT, 2: no recurrent infection after treatment, and 3: recurring infection after tube placement.

data from the first visit to the otolaryngology clinic (patient evaluation and preoperative consultation). Cross-sectional OCT shows a bright, scattering biofilm structure (green arrow) affixed to the medial mucosal surface of the TM, as well as evidence of suspended, optically scattering particles in the MEC, indicative of an MEE (white arrow, dashed). The corresponding video otoscope surface image shows a dull and relatively featureless TM (inset). Here, and in subsequent figures, yellow and green bars denote the location and thickness of the TM and biofilm, respectively, as determined using a previous method. ${ }^{21}$ Figure 3(b) shows intraoperative image data from this same subject and ear (second visit) prior to myringotomy, aspiration of the MEC, and TT placement. OCT imaging confirmed the persistence of infectionrelated image-based features, including the biofilm (green arrow, green line) and MEE (white arrow, dashed) imaged at the consultation visit, although scanned at a slightly different TM location within the light reflex region.

Approximately one month later, the same subject was imaged at the third visit (postoperative follow-up) following standard postsurgical care. Here, the microstructural effects of surgical intervention are visualized. In Fig. 3(c), the MEC is free of any MEE and biofilm. A portion of the TT (blue arrows, dashed) deeper inside the MEC is visualized. The TM (yellow 

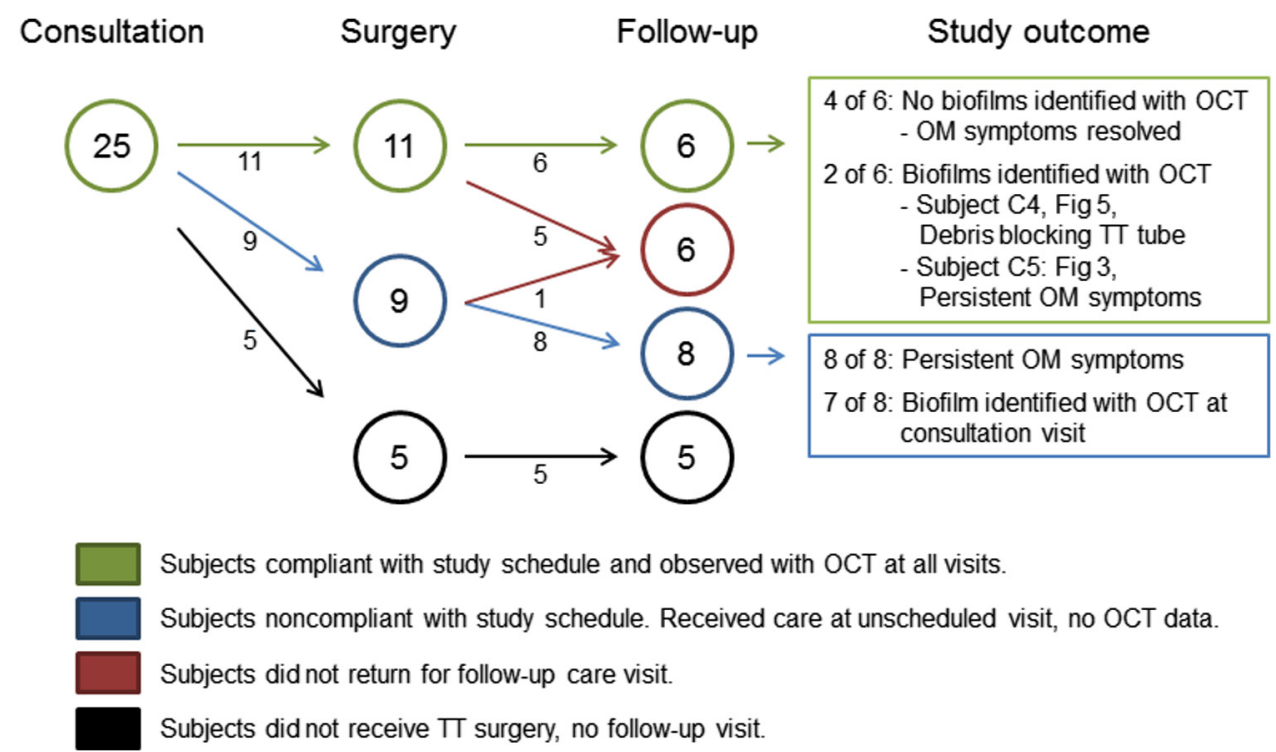

Fig. 2 Graphical breakdown of subjects enrolled in this study. On the day of preoperative consultation, 23 out of 25 subjects had a biofilm identified with OCT. On the day of surgery, 11 subjects were observed with OCT, and 10 out of 11 subjects had biofilms identified with OCT prior to surgical intervention. On the final day of follow-up, 6 subjects returned for OCT imaging. These subjects comprised the complete group identified in Table 1, with OCT data and clinical reports available from each time point. Two out of 6 subjects had additional structures visible in OCT images at follow-up, and subject C5 reported persistent OM symptoms. Four out of 6 subjects had no reported OM symptoms and had no biofilm identified with OCT. Only partial data were able to be collected from the remaining 19 subjects who were noncompliant with the study schedule. The partial group included OCT data from the day of consultation and patient reports if/when care was received at an unscheduled visit. Eight out of 19 subjects that returned for unscheduled visits reported persistent OM symptoms, and 7 out of 8 of these subjects had a biofilm previously identified at the day of consultation. Six out of 19 subjects did not return for followup care after receiving TT surgery, and 5 out of 19 did not receive TT surgery or have a follow-up visit scheduled. OM: otitis media and TT: tympanostomy tube.

line) appears thicker due to the presence of residual earwax (orange arrows, orange line) on the outer surface of the TM and because the image was acquired closer to the umbo, where the $\mathrm{TM}$ is naturally thicker. ${ }^{54}$ The surface image (inset) shows that some transparency has returned to the TM. Otoscopic metrics provide visual confirmation of surgical outcomes and, in this case, indicate a reduction in inflammation and a fluid-free middle-ear space compared to presurgery time points. The TT is observed in place and functionally venting the MEC. ${ }^{47,55}$ At the end of the records-based observation period, this subject did not report any symptoms of OM recurrence.

When an infection occurred after postoperative follow-up or perhaps when surgery was ineffective at clearing infectionrelated components, additional biofilm- or MEE-related structures were observed affixed to the TM and/or within the MEC, respectively. Figure 4 highlights subject $\mathrm{C} 5$, who was presented with a history of recurrent episodes of acute OM that persisted after TT placement. Visible biofilm structures detected in OCT cross-sectional images persisted throughout all observation time points, including pre-TT placement [Figs. 4(a) and 4(b), green arrows] and post-TT placement [Fig. 4(c), green arrows]. Yellow and green bars indicate the location and thickness of the TM and biofilm, respectively. Longer-term follow-up was possible given a recurrence of the infection in this subject, who required additional outpatient visits. Approximately six months after the postoperative visit, a patchy or spatially inconsistent biofilm was observed affixed to the TM [Fig. 4(d)], while in some regions no biofilm was present [Fig. 4(e)]. The identification of both seemingly normal and pathological regions in these OCT images highlighted here was unexpected and found in only one ear from this one subject. However, this finding demonstrates the complex dynamics of biofilm formation in OM and regression after treatment, indicating that further investigation and longitudinal tracking at more time points may better capture these processes. The other ear of this subject and all other study subjects had consistent findings from each time point. No digital otoscopy image was available due to patient discomfort. An indisputable and unavoidable limitation in studies involving human subjects, especially those in active clinical and surgical settings, is the priority of patient needs and well-being over data collection, which in some cases presented a challenge for acquiring complete datasets. This subject was provided with antibiotics based on the physical exam and was scheduled for re-evaluation at a later date.

Available data from the $\mathrm{P}$ dataset was typically limited to the first consultation visit and included OCT images, digital otoscopy images, and patient history. Imaging data from subsequent visits were not available and were lost to follow-up, a common problem in otolaryngology, ${ }^{52}$ as imaging subjects in this observational study were free to seek, reschedule, or not seek care as per their wishes. Almost all subjects in the $\mathrm{P}$ group had a reported history of recurrent OM $(18 / 19,95 \%)$ and mostly $(17 / 19,90 \%)$ were found with OCT to have biofilm-like structures affixed to the TM during the first visit, as noted in our previous studies. A total of 20 out of 25 subjects $(80 \%)$ underwent TT surgery. However, 8 out of $25(32 \%)$ 

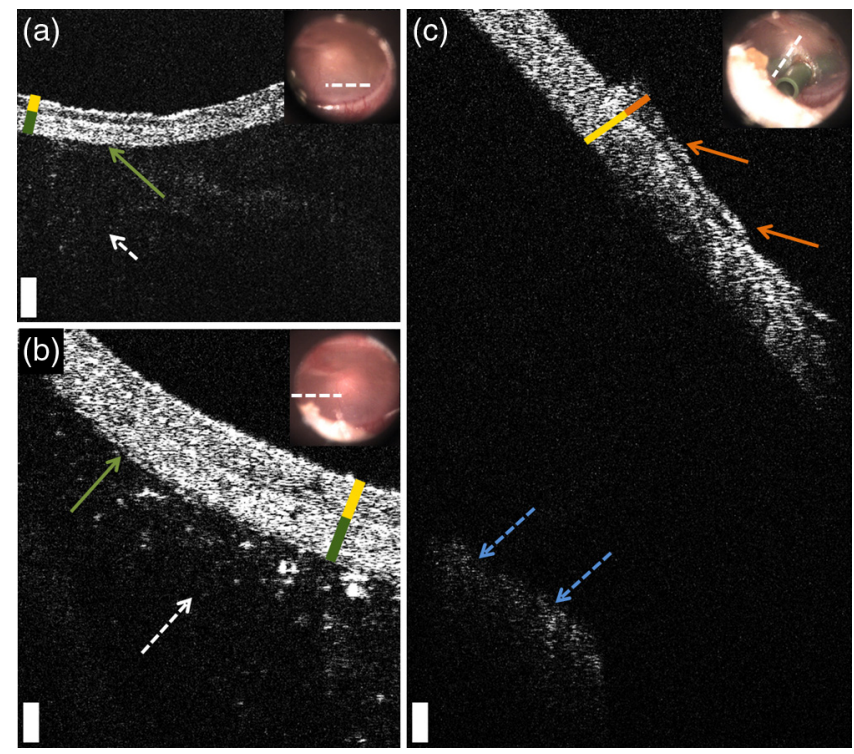

Fig. 3 Representative longitudinal interventional data from subject C3. Cross-sectional OCT images were acquired at (a) the preoperative evaluation and surgical consultation, (b) the day of surgery prior to myringotomy, and (c) at the postoperative follow-up. Arrows denote the additional infection-related components. Digital otoscopy images of the ear taken at the surgical consultation and follow-up are shown as insets, where white-dashed lines denote where the cross-sectional OCT images were acquired. ( $a$ and b) Biofilms (green arrows) are visualized as being affixed to the TM, and effusions (containing many point-like optically scattering particles in suspension) (white arrows, dashed) are visualized within the MEC. (c) Images from the follow-up show a cleared MEC with an image acquired near the umbo, a thicker region of the TM when compared to the light reflex. Earwax is also present on the outer surface of the TM (orange arrows, orange line), and the TT is visible deeper within the MEC (blue arrows, dashed). Surgical intervention appears to have cleared any biofilm and effusion components visible under OCT from this MEC. Scale bars represent $100 \mu \mathrm{m}$ in depth. Yellow, green, and orange bars indicate the positions and thicknesses of the TM, biofilm, and earwax, respectively.

subjects reported recurrence of infection during the postoperative observation period, which may have resulted from local recurrence of the original infection and biofilm or from a new, discrete OM infection. While TT surgery was performed depending on the clinical circumstances of each patient, it is notable that 3 out of $19(16 \%)$ in our study had a history of multiple TT surgeries, with 2 of these subjects having biofilm-related structures present in OCT images. Additional figures are presented in the Appendix that demonstrates the diverse presentation of recurrent or chronic OM encountered in this study.

\section{Discussion}

Additional infection-related structures were initially observed in OCT images from most subjects $(92 \%, 23 / 25)$ diagnosed with recurrent or chronic OM and scheduled to undergo TT surgery, as per their standard-of-care. Through record-based follow-up of all subjects, surgical intervention was effective at clearing the infection and reducing or eliminating symptoms in 15 out of 20 subjects $(75 \%)$ that received TT surgery. If observed with OCT, image analysis confirmed the absence of a middle-ear biofilm in these subjects. However, 5 out of 20 subjects (25\%) that received surgery had persistent clinical symptoms of OM infection following TT surgery, and if observed with OCT, infection- related structures were present. These structures were similarly identified in subjects that had multiple surgical interventions and had returned for repeated treatments. Therefore, based on OCT imaging results, if a subject did not respond to surgical intervention, there was likely an insufficient clearance of infectious material from the MEC or a recurrence of the bacterial population and subsequent biofilm regrowth. A postsurgical OCT confirmation of the absence of a biofilm could then perhaps serve as a secondary diagnostic indicator or predictor for long-term success of the surgical intervention. A future study could similarly explore the effectiveness of pharmacological interventions to clear symptoms of OM in subjects identified with a middleear biofilm.

Surgical intervention, in this case myringotomy and TT placement, results in both short- and long-term effects that influence several changes in an infected MEC. With myringotomy, a renewed immune and healing response is stimulated ${ }^{56}$ along with aspiration of the MEC and insertion of a TT. These interventions aid in clearing middle-ear fluid and provide a means of aerating the MEC. When considered in the context of biofilm-related infections, mechanical clearance and aspiration of the MEC disturb any biofilm and its growth environment both structurally and biochemically. Ototopical drops placed into the MEC also provide a higher and more effective concentration of antibiotics directly to the site of infection ${ }^{57,58}$ when compared to oral antibiotics. ${ }^{59}$ Successful clearance of the MEC and reduction of OM-related symptoms may indicate these structures are cleared by a combination of these factors.

Regardless of any potential new diagnostic information gained with OCT, considering recurrent or chronic OM as a biofilm-mediated disease would also require investigating new or re-evaluating existing treatment options. ${ }^{60,61}$ Methods that attempt to noninvasively weaken, disrupt, or clear a biofilm ${ }^{62-64}$ could be employed before invasive means are considered or even employed in conjunction with surgical treatment for a more aggressive approach. However, if TT placement is found to be one of the most effective therapeutic interventions to treat biofilm-derived recurrent or chronic OM, then it will be necessary to weigh the related risks and outcomes associated with presumably more patients receiving tubes at a younger age. Serious consideration should be given to the fact that a majority of patients tend to outgrow complications and/or susceptibility to recurrent $\mathrm{OM}$, perhaps due to improved function and increased diameter of the Eustachian tube with age ${ }^{65}$ or improved immune function with age. ${ }^{66}$ Conversely, it is also important to consider that many children are unnecessarily hearing impaired during this time, which delays learning and speech development at a crucial age in young subjects. Further investigation is undeniably necessary to observe broader subject population demographics, as well as biochemical and genetic factors, to more fully understand the dominant factors of middle-ear disease pathogenesis, as well as any physical or secondary indicators that can be used for diagnosis and monitoring. In this regard, tracking the reduction or clearance of biofilm biomass with OCT after surgical or pharmacological treatment may provide further insight into mechanisms for effective treatment of OM.

To suggest any potential use of this OCT data for clinicians, further study is still needed and should include a randomized clinical trial with a larger subject population and a wider geographic and demographic distribution to capture a broader view of OM. With this longer-term study, diagnostic features in OCT images may be identified that predict a need for surgical 

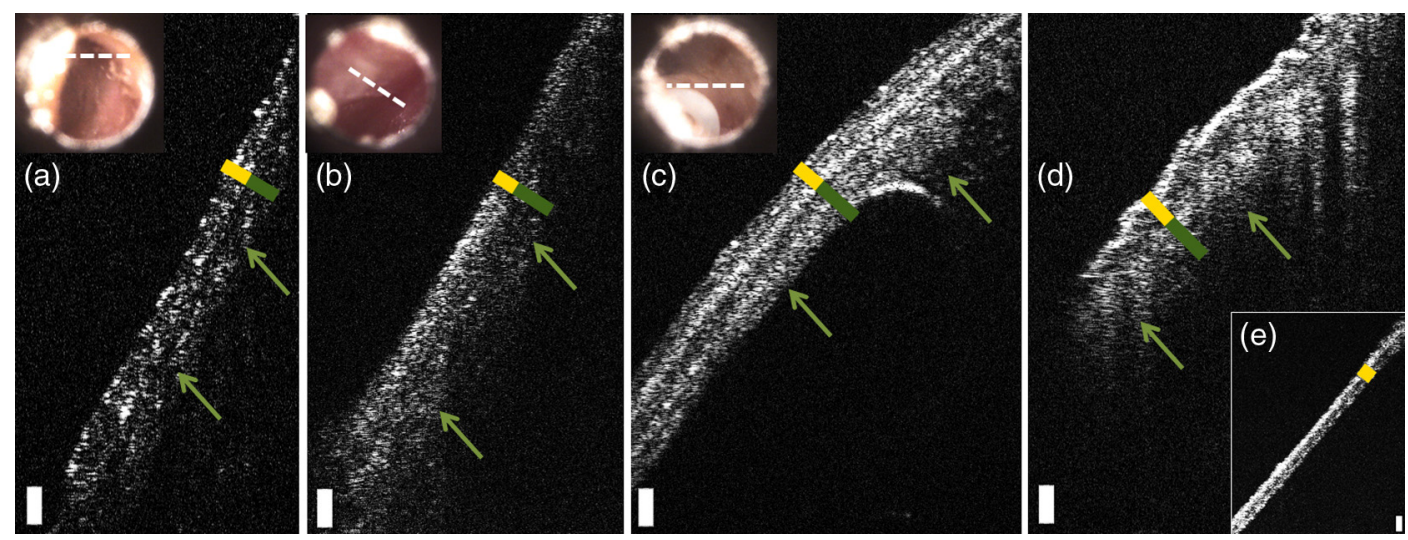

Fig. 4 Longitudinal imaging of the TM and infection-related components from study participant C5. Cross-sectional OCT images of the middle ear are shown at various time points in the progression of infection and treatment of subject C5, along with inset digital otoscopy images of the ear: (a) patient evaluation and surgical consultation, (b) day of surgery prior to myringotomy, (c) postoperative follow-up, and ( $\mathrm{d}$ and $\mathrm{e}$ ) at later, long-term follow-up visits. Arrows denote persistent additional, infection-related structures. Digital otoscopy imaging for (d) and (e) was not possible due to patient temperament and discomfort. Subject C5 received TTs to treat recurrent acute otitis media (AOM) and hearing loss. After a one-month recovery from TT placement (c), additional middle-ear structures were visible (green arrows). However, (d) and (e) show two images from long-term follow-up exams roughly six months after (c), after the subject revisited both the primary care office and specialists clinic for OMrelated complaints. While (d) shows additional structure (green arrows), (e) appears normal, perhaps indicating a persistent, but spatially inconsistent and patchy middle-ear biofilm within the light reflex region of this subject. Scale bars represent $100 \mu \mathrm{m}$ in depth. Yellow and green bars indicate the position and thickness of the TM and biofilm, respectively.

intervention. This may allow for a quantitative and evidencedbased referral to surgery at earlier time points in the pathogenesis of chronic OM, potentially shortening the duration of disease symptoms and related morbidity prior to treatment and successful resolution. In this regard, OCT-based treatment guidelines for OM will need to be proposed, established, and evaluated, as was done for clinical cardiovascular ${ }^{67}$ OCT and clinical ophthalmic ${ }^{68}$ OCT clinical applications.

There are several limitations in this initial observational study. With the spectral-domain OCT system used in these investigations, it was not technically possible to image deeper to the medial mucosal surfaces of the MEC. However, sweptsource OCT systems can provide deeper imaging to over a centimeter, ${ }^{69}$ which can provide additional information on the location, extent, and severity of any middle-ear biofilm or MEE simultaneously on both the interior mucosal surface of the TM as well as the mucosal surface throughout the MEC. While the light reflex region of the TM was interrogated in this study and used as a consistent anatomical landmark for longitudinal image collection, the remaining areas of the TM may provide further information related to the biofilm or other middle-ear diseases. Techniques for rapidly generating three-dimensional images of the entire TM, while minimizing motion artifacts and coregistering these datasets among longitudinal visits are in development. The presence of specific otopathogens may ultimately govern whether surgery is effective. ${ }^{70}$ However, OCT is not sensitive to specific bacterial species. Ongoing research in our group is investigating the use of Raman spectroscopy for determining microbial content of the middle ear, ${ }^{71}$ which has previously been used to differentiate bacterial strains in culture. ${ }^{72}$

Finally, additional studies are needed to verify longer-term outcomes of study participants with OCT and the clinical relevance of the presence or absence of a middle-ear biofilm in patients with chronic OM. Another ongoing study is aimed at physically sampling, identifying, and verifying the infectionrelated structures and microbiological content that has been observed on the TM with OCT. Results will help to better understand and correlate the image-based findings with the presence of biofilms that were previously detected on middle-ear mucosa during chronic OM. ${ }^{26}$

\section{Conclusion}

In this study, a broad subject population was prospectively observed using OCT to assess the efficacy of surgical intervention to clear OM-related middle-ear fluid or biofilms and associated clinical symptoms. OCT image findings suggest that surgical intervention, consisting of myringotomy followed by TT placement, provides a means to clear the middle ear of infection-related biofilm structures and components. OCT shows promise to provide a clinically viable diagnostic and monitoring platform capable of providing physicians with new image-based information about the disease state of the middle ear. With further investigation, OCT image features may aid in the diagnosis and management of OM, especially when interpreted alongside physical exam, otoscopy findings, and patient history.

\section{Appendix}

Here, additional data are presented that survey the wide range of clinical presentations of chronic or recurrent OM observed in this study.

Figure 5 shows representative data from this group (subject P3). This subject presented with both moderate conductive hearing loss and an identified MEE in each ear. This subject was observed at both the first [Fig. 5(a)] and second [Fig. 5(b)] visits. Yellow and green bars indicate the location and thickness of the TM and biofilm, respectively. At both time points, additional infection-related structures were clearly visible within the MEC. 

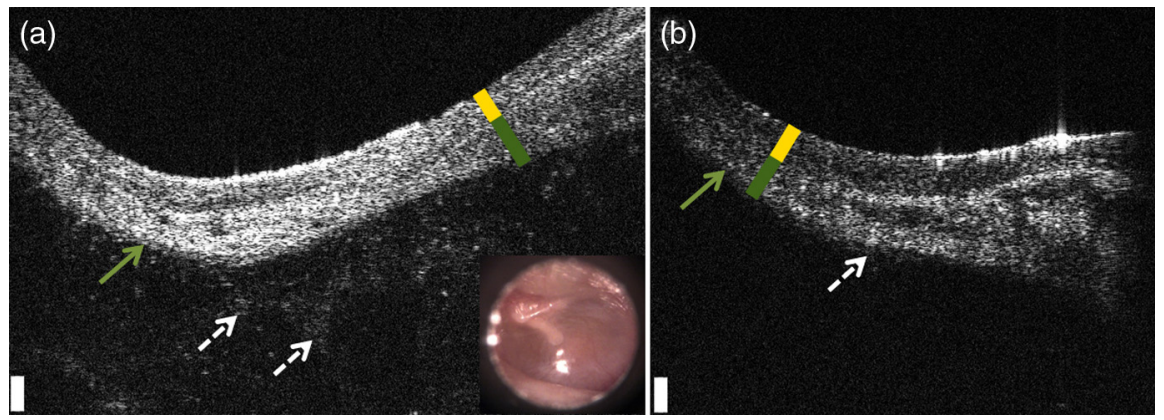

Fig. 5 Representative data from the partial $(P)$ group. Subject $P 3$ presented with both moderate conductive hearing loss and identified MEEs in each ear. Cross-sectional OCT data and inset digital otoscopy images of the ear are shown, if available, (a) from the patient evaluation and surgical consultation and (b) from the day of surgery prior to myringotomy. Arrows denote persistent additional, infectionrelated structures (green arrows: biofilm, white arrows and dashed: MEEs). The subject did not return for postsurgical follow-up care at the originally scheduled time, so only notes from the physical exam were available. Scale bars represent $100 \mu \mathrm{m}$ in depth. Yellow and green bars indicate the position and thickness of the TM and biofilm, respectively.

This subject, like others in the $\mathrm{P}$ group, was noncompliant with scheduled follow-up exam times, and therefore, only information from the physical exam was available from the third visit.

Figure 6 shows data from subject $\mathrm{C} 4$, diagnosed with persistent or frequent recurrent acute OM and ETD. Figure 6(a), acquired during the preoperative visit, shows an overall thickened structure comprised of the TM and attached biofilm. In this image, delineation of the TM from the biofilm can be challenging, likely because of refractive index matching occurring among these structures. Figure 6(b), acquired intraoperatively immediately prior to TT placement, shows that the biofilm is no longer present and was replaced by an MEE (white arrows). When imaged at the postsurgical follow-up visit [Fig. 6(c)], additional structures (green arrows) were found to be affixed to the TM. Although this subject had no reported symptoms of recurrent infection during the study period, the obstructed TT, as visualized in the inset digital otoscopy image, would have provided limited aeration and drainage of the MEC. This blockage likely prevented complete clearance of the middle-ear space.

Figure 7 presents images from subject C6, who was originally presented with ETD and recurrent acute OM. When viewed at the postsurgical follow-up, the cross-sectional OCT images showed a TT within a cleared MEC. This again suggests that surgical intervention successfully clears the middle-ear space of biofilm-related structures. Another benefit of OCT is also indirectly highlighted in the digital otoscopy image of the TM. Most of the ear canal in this subject was occluded with cerumen, which makes any accurate observation or diagnosis using standard otoscopy prohibitive without first cleaning the ear canal to properly view the entire TM. However, the OCT beam only requires a small, clear direct line-of-sight imaging path and can propagate through small gaps in the cerumen within the tortuous ear canal.

Subjects with repeated TT placement surgeries often present with different image-based TM features than those undergoing their first procedure. For instance, depending on the duration since the last TT surgery, thinning in the fibrous layer of the TM may be evident, or even a residual opening from an extruded TT may often still be visible in the TM. Figure 8 shows such a case from subject P14, who presented with persistent OME after a recent TT surgery. When examined, a residual perforation in the TM from a previous TT was visible in the otoscopy image.
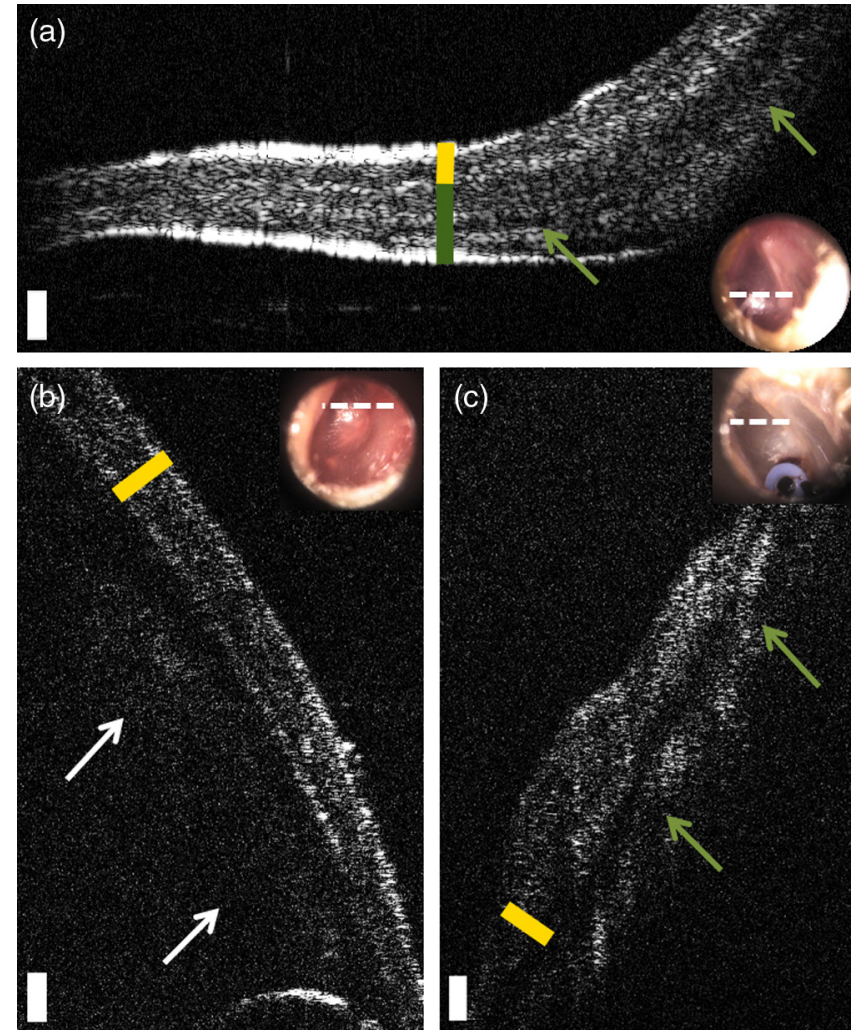

Fig. 6 A potentially ineffective surgical intervention from subject C4. Cross-sectional OCT images of the (a) patient evaluation and surgical consultation, (b) day of surgery prior to myringotomy, and (c) postoperative follow-up are shown along with digital otoscopy images (inset). White-dashed lines on the digital otoscopy images denote where the cross-sectional OCT images were taken. The image in (a) shows an overall thickened TM, with a suspected biofilm-like structure affixed on the mucosal surface of the TM (green arrows). (b) At the day of surgery, this biofilm-like structure is no longer present but replaced by a low-scattering effusion (white arrows). (c) During the postoperative follow-up, the tissue appears thickened and possibly inflamed or scarred, with additional middle-ear structures affixed to the TM (green arrows). In this specific case, the TT was found to be clogged with dark brown debris, likely dried discharge from the middle ear. The blockage likely prevented aeration and drainage from the MEC and complete clearance of the middle-ear space. Scale bars represent $100 \mu \mathrm{m}$ in depth. Yellow and green bars indicate the position and thickness of the TM and biofilm, respectively. 

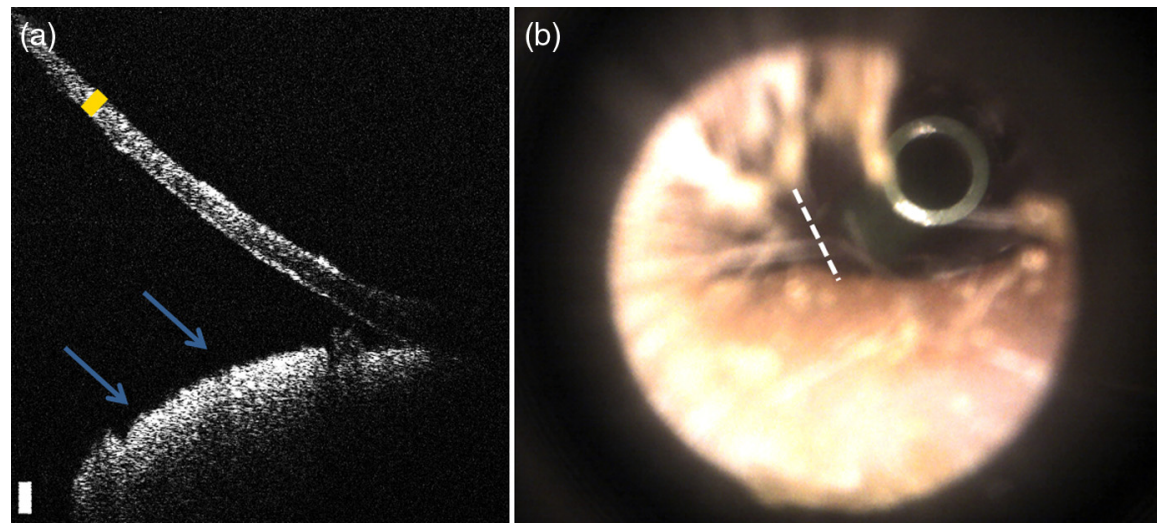

Fig. 7 Cross-sectional OCT image and digital otoscopy image from subject $\mathrm{C}$, who was originally presented with ETD and recurrent acute OM. Viewed at the postsurgical follow-up, imaging results show a cleared middle-ear space. (a) OCT image of a TM with the TT visible below within the MEC (blue arrows). (b) Digital otoscopy image showing the ear canal and TM partially occluded by cerumen. Although cerumen prevents direct visualization of the TM, the OCT beam can navigate small openings in the cerumen and successfully image the TM and MEC. OCT image taken near the white-dashed line. Scale bar represents $100 \mu \mathrm{m}$ in depth. The yellow bar denotes the position and thickness of the TM.

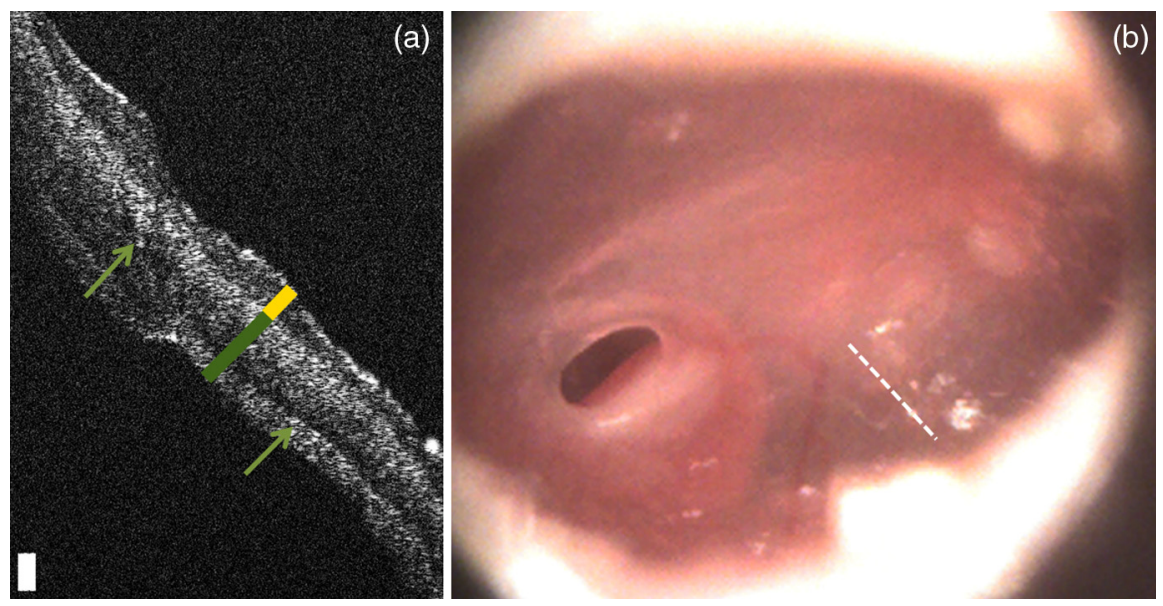

Fig. 8 Cross-sectional OCT image and digital otoscopy image from subject P14, who was presented with persistent OME and had previously received a TT placement. (a) OCT image of a thinned irregular TM and adherent biofilm (green arrows). (b) Digital otoscopy image shows a previous perforation from a prior TT placement. OCT image was taken at the location indicated by the white-dashed line in (b). Scale bar represents $100 \mu \mathrm{m}$ in depth.

OCT of the TM showed a thinned, irregular TM and a thick, adherent, persistent biofilm (green arrows).

\section{Disclosures}

Authors S.A.B., R.L.S., R.M.N., and M.A.N. disclose a financial interest in PhotoniCare, Inc., which is commercializing OCT for imaging the ear. The other authors have no funding, financial relationships, or conflicts of interest to disclose.

\section{Acknowledgments}

The authors would like to acknowledge the research staff at Carle Foundation Hospital, especially Deveine Toney, and the nursing staff at both the Carle Expanding Children's Hearing Opportunities Center and the Carle Ambulatory Surgery Center, for their assistance during the examinations and surgeries. This work was funded by a National Institutes of Health Bioengineering Research Partnership (No. R01EB013723, S.A.B.).
Additional information can be found at http://biophotonics. illinois.edu.

\section{References}

1. K. Ramakrishnan, R. Sparks, and W. Berryhill, "Diagnosis and treatment of otitis media," Am. Fam. Physician 76(11), 1650-1658 (2007).

2. R. Linsk et al., "Otitis media guideline for clinical care," 2007, University of Michigan Health System, Ann Arbor, Michigan, http:// ocpd.med.umich.edu/sites/default/files/guidelines/OM.pdf (May 2015).

3. P. Shekelle et al., "Diagnosis, natural history, and late effects of otitis media with effusion," Evidence Report/Technology Assessment \#55 (Prepared by Southern California Evidence-based Practice Center under Contract No 290-97-0001, Task Order No. 4), AHRQ \#03E023, Agency for Healthcare Research and Quality, Rockville, Maryland (2003).

4. E. Morris et al., "Development and validation of a novel ear simulator to teach pneumatic otoscopy," Simul. Healthcare 7(1), 22-26 (2012).

5. K. K. Helenius et al., "Tympanometry in discrimination of otoscopic diagnoses in young ambulatory children," Pediatr. Infect. Dis. J. 31(10), 1003-1006 (2012). 
6. C. T. Nguyen et al., "Noninvasive in vivo optical detection of biofilm in the human middle ear," Proc. Natl. Acad. Sci. U. S. A. 109(24), 95299535 (2012).

7. C. T. Nguyen et al., "Non-invasive optical interferometry for the assessment of biofilm growth in the middle ear," Biomed. Opt. Express 1(4), 1104-1116 (2010).

8. S. Keyhani et al., "Overuse of tympanostomy tubes in New York metropolitan area: evidence from five hospital cohort," Br. Med. J. 337, a1607 (2008).

9. I. F. Wallace et al., "Surgical treatments for otitis media with effusion: a systematic review," Pediatrics 133(2), 296-311 (2014).

10. M. A. Steinman et al., "Variation in outpatient antibiotic prescribing in the United States," Am. J. Managed Care 15(12), 861-868 (2009).

11. J. L. Lyon et al., "Variation in the diagnosis of upper respiratory tract infections and otitis media in an urgent medical care practice," Arch. Fam. Med. 7(3), 249-254 (1998).

12. T. Marom et al., "Trends in otitis media-related health care use in the United States, 2001-2011," JAMA Pediatr. 168(1), 68-75 (2014).

13. N. H. Cho et al., "In vivo imaging of middle-ear and inner-ear microstructures of a mouse guided by SD-OCT combined with a surgical microscope," Opt. Express 22(8), 8985-8995 (2014).

14. S. J. Erickson-Bhatt et al., "Real-time imaging of the resection bed using a handheld probe to reduce incidence of microscopic positive margins in cancer surgery," Cancer Res. 75(18), 3706-3712 (2015).

15. J. L. Lin et al., "Real-time subglottic stenosis imaging using optical coherence tomography in the rabbit," JAMA Otolaryngol. Head Neck Surg. 139(5), 502-509 (2013).

16. S. Van der Jeught et al., "Full-field thickness distribution of human tympanic membrane obtained with optical coherence tomography," J. Assoc. Res. Otolaryngol. 14(4), $483-494$ (2013).

17. J. Park et al., "Investigation of middle ear anatomy and function with combined video otoscopy-phase sensitive OCT," Biomed. Opt. Express 7(2), 238-250 (2016).

18. A. M. Karamzadeh et al., "Characterization of submucosal lesions using optical coherence tomography in the rabbit subglottis," Arch. Otolaryngol. Head Neck Surg. 131(6), 499-504 (2005).

19. C. Pitris et al., "High-resolution imaging of the middle ear with optical coherence tomography: a feasibility study," Arch. Otolaryngol. Head Neck Surg. 127(6), 637-642 (2001).

20. E. J. Chaney, C. T. Nguyen, and S. A. Boppart, "Novel method for noninvasive induction of a middle-ear biofilm in the rat," Vaccine 29(8), 1628-1633 (2011).

21. G. L. Monroy et al., "Noninvasive depth-resolved optical measurements of the tympanic membrane and middle ear for differentiating otitis media," Laryngoscope 125(8), E276-E282 (2015).

22. C. T. Nguyen et al., "Investigation of bacterial biofilm in the human middle ear using optical coherence tomography and acoustic measurements," Hear. Res. 301, 193-200 (2013).

23. R. L. Shelton et al., "Optical coherence tomography for advanced screening in the primary care office," J. Biophotonics 7(7), 525-533 (2014).

24. G. L. Monroy et al., "Non-invasive optical assessment of viscosity of middle ear effusions in otitis media," J. Biophotonics 10(3), 394-403 (2017).

25. H. R. Djalilian et al., "Imaging the human tympanic membrane using optical coherence tomography in vivo," Otol. Neurotol. 29(8), 10911094 (2008).

26. L. Hall-Stoodley et al., "Direct detection of bacterial biofilms on the middle-ear mucosa of children with chronic otitis media," J. Am. Med. Assoc. 296(2), 202-211 (2006).

27. J. M. Coticchia et al., "New paradigms in the pathogenesis of otitis media in children," Front. Pediatr. 1, 52 (2013).

28. M. Daniel et al., "Bacterial involvement in otitis media with effusion," Int. J. Pediatr. Otorhinolaryngol. 76(10), 1416-1422 (2012).

29. L. Nistico et al., "Adenoid reservoir for pathogenic biofilm bacteria," J. Clin. Microbiol. 49(4), 1411-1420 (2011).

30. L. Tóth et al., "Biofilm detection in chronic rhinosinusitis by combined application of hematoxylin-eosin and gram staining," Eur. Arch. OtoRhino-Laryngol. 268(10), 1455-1462 (2011).

31. M. Hoa et al., "Biofilms and chronic otitis media: an initial exploration into the role of biofilms in the pathogenesis of chronic otitis media," Am. J. Otolaryngol. 31(4), 241-245 (2010).
32. K. E. D. Weimer et al., "Coinfection with Haemophilus influenzae promotes pneumococcal biofilm formation during experimental otitis media and impedes the progression of pneumococcal disease," $J$. Infect. Dis. 202(7), 1068-1075 (2010).

33. R. E. Kania et al., "Characterization of mucosal biofilms on human adenoid tissues," Laryngoscope 118(1), 128-134 (2008).

34. R. B. Thornton et al., "Multi-species bacterial biofilm and intracellular infection in otitis media," BMC Pediatr. 11, 94 (2011).

35. R. N. Masyuko et al., "Spatial organization of Pseudomonas aeruginosa biofilms probed by combined matrix-assisted laser desorption ionization mass spectrometry and confocal Raman microscopy," Analyst 139(22), 5700-5708 (2014).

36. G. Zhao et al., "Biofilms and inflammation in chronic wounds," $A d v$. Wound Care 2(7), 389-399 (2013).

37. C. R. Arciola et al., "Biofilm formation in Staphylococcus implant infections. A review of molecular mechanisms and implications for biofilm-resistant materials," Biomaterials 33(26), 5967-5982 (2012).

38. H.-S. Joo and M. Otto, "Molecular basis of in-vivo biofilm formation by bacterial pathogens," Chem. Biol. 19(12), 1503-1513 (2012).

39. P. Dwivedi et al., "Role of Bcrl-activated genes Hwp1 and Hyr1 in Candida albicans oral mucosal biofilms and neutrophil evasion," PLoS One 6(1), e16218 (2011).

40. T. Bjarnsholt, O. Ciofu, and N. Hoiby, "Pseudomonas aeruginosa biofilms in cystic fibrosis," Future Microbiol. 5(11), 1663-1674 (2010).

41. M. Martinez-Medina et al., "Biofilm formation as a novel phenotypic feature of adherent invasive Escherichia coli (AIEC)," BioMed Cent. Microbiol. 9, 202 (2009).

42. D. D. Rhoads, R. D. Wolcott, and S. L. Percival, "Biofilms in wounds: management strategies," J. Wound Care 17(11), 502-508 (2008).

43. D. Monroe, "Looking for chinks in the armor of bacterial biofilms," PLoS Biol. 5(11), e307 (2007).

44. J. M. Coticchia, D. Cohen, and L. Sachdeva, "Grand challenges in pediatric otolaryngology," Front. Pediatr. 1, 10 (2013).

45. A. S. Lieberthal et al., "The diagnosis and management of acute otitis media," Pediatrics 131(3), e964-e999 (2013).

46. R. M. Rosenfeld et al., "Clinical practice guideline: otitis media with effusion (update)," Otolaryngol. Head Neck Surg. 154(Suppl. 1), S1-S41 (2016).

47. R. M. Rosenfeld et al., "Clinical practice guideline: tympanostomy tubes in children-executive summary," Otolaryngol. Head Neck Surg. 149(1), 8-16 (2013).

48. ANSI, "Z136.1—safe use of lasers," Laser Institute of America, Orlando, Florida (2014).

49. Z. Hubler et al., "Real-time automated thickness measurement of the in vivo human tympanic membrane using optical coherence tomography," Quant. Imaging Med. Surg. 5(1), 69-77 (2015).

50. S. R. Schwartz et al., "Clinical practice guideline (update): earwax (cerumen impaction)," Otolaryngol. Head Neck Surg. 156(Suppl. 1), S1-S29 (2017).

51. E. L. Yang et al., "Economic and demographic characteristics of cerumen extraction claims to medicare," JAMA Otolaryngol. Head Neck Surg. 142(2), 157-161 (2016).

52. R. Kao, D. J. Kirse, and A. K. Evans, "Compliance with recommendations for tympanostomy tube follow-up: patient characteristics," Otolaryngol. Head Neck Surg. 151(3), 489-495 (2014).

53. C. M. Song et al., "Factors affecting the extrusion rate of ventilation tubes," Clin. Exp. Otorhinolaryngol. 3(2), 70-75 (2010).

54. P. Pande et al., "A mosaicking approach for in vivo thickness mapping of the human tympanic membrane using low coherence interferometry," J. Assoc. Res. Otolaryngol. 17(5), 403-416 (2016).

55. American Academy of Pediatrics, "Follow-up management of children with tympanostomy tubes," Pediatrics 109, 328-329 (2002).

56. M. M. D. Araújo et al., "Spontaneous healing of the tympanic membrane after traumatic perforation in rats," Braz. J. Otorhinolaryngol. 80, 330-338 (2014).

57. S. Couzos et al., "Effectiveness of ototopical antibiotics for chronic suppurative otitis media in Aboriginal children: a community-based, multicenter, double-blind randomised controlled trial," Med. J. Aust. 179(4), 185-190 (2003).

58. S. M. Wintermeyer, M. C. Hart, and M. C. Nahata, "Efficacy of ototopical ciprofloxacin in pediatric patients with otorrhea," Otolaryngol. Head Neck Surg. 116(4), 450-453 (1997). 
59. G. S. Renukananda, U. P. Santosh, and N. M. George, "Topical vs combination ciprofloxacin in the management of discharging chronic suppurative otitis media," J. Clin. Diagn. Res. 8(6), KC01-KC04 (2014).

60. H. Wu et al., "Strategies for combating bacterial biofilm infections," Int. J. Oral Sci. 7(1), 1-7 (2015).

61. M. N. Hurley, M. Cámara, and A. R. Smyth, "Novel approaches to the treatment of Pseudomonas aeruginosa infections in cystic fibrosis," Eur. Respir. J. 40(4), 1014-1023 (2012).

62. M. A. D. De Rienzo et al., "Pseudomonas aeruginosa biofilm disruption using microbial surfactants," J. Appl. Microbiol. 120(4), 868-876 (2016).

63. M. Zakrewsky et al., "Ionic liquids as a class of materials for transdermal delivery and pathogen neutralization," Proc. Natl. Acad. Sci. U. S. A. 111(37), 13313-13318 (2014).

64. R. B. Thornton et al., "Neutrophil extracellular traps and bacterial biofilms in middle ear effusion of children with recurrent acute otitis media—a potential treatment target," PLoS One 8(2), e53837 (2013).

65. M. Kitajiri, I. Sando, and T. Takahara, "Postnatal development of the Eustachian tube and its surrounding structures preliminary study," Ann. Otol. Rhinol. Laryngol. 96(2), 191-198 (1987).

66. S. K. Sharma and M. E. Pichichero, "Cellular immune response in young children accounts for recurrent acute otitis media," Curr. Allergy Asthma Rep. 13(5), 495-500 (2013).

67. H. G. Bezerra et al., "Intracoronary optical coherence tomography: a comprehensive review-clinical and research applications," JACC Cardiovasc. Interventions 2(11), 1035-1046 (2009).

68. R. S. Jonnal et al., "A review of adaptive optics optical coherence tomography: technical advances, scientific applications, and the future," Invest. Ophthalmol. Visual Sci. 57(9), OCT51-OCT68 (2016).

69. M. Bonesi et al., "Akinetic all-semiconductor programmable sweptsource at $1550 \mathrm{~nm}$ and $1310 \mathrm{~nm}$ with centimeters coherence length," Opt. Express 22(3), 2632-2655 (2014).

70. R. Osgood et al., "Effect of pH and oxygen on biofilm formation in acute otitis media associated NTHi clinical isolates," Laryngoscope 125(9), 2204-2208 (2015).

71. Y. Zhao et al., "Rapid diagnosis and differentiation of microbial pathogens in otitis media with a combined Raman spectroscopy and low-coherence interferometry probe: toward in vivo implementation," J. Biomed. Opt. 21(10), 107005 (2016).

72. S. Stöckel et al., "The application of Raman spectroscopy for the detection and identification of microorganisms," J. Raman Spectrosc. 47(1), 89-109 (2016).

Guillermo L. Monroy is currently a graduate research fellow (PhD candidate) in the Biophotonics Imaging Laboratory, working under the guidance of Professor Stephen Boppart. He received his BS degree in computer engineering in 2011 and continued for his MS in bioengineering at the University of Illinois at Urbana-Champaign in 2013. His current academic interests include the integration of advanced biomedical instrumentation and imaging techniques to improve diagnosis and treatment in healthcare.

Paritosh Pande received his BTech (hons) degree in electrical engineering and his MTech degree in instrumentation engineering, both from the Indian Institute of Technology, Kharagpur, India, in 2008, and his $\mathrm{PhD}$ in biomedical engineering from Texas A\&M University, College Station, Texas, in 2014. His research interests include biomedical signal and image processing, pattern recognition, and the development of optical imaging technologies for biomedical diagnostics.
Ryan M. Nolan received his MEng degree in biomedical engineering from Cornell University and then joined the Biophotonics Imaging Lab, the University of Illinois at Urbana-Champaign as an imaging research specialist. Currently, he is with PhotoniCare, a medical device company seeking to improve the diagnosis and management of middleear disease.

Ryan L. Shelton received his $\mathrm{PhD}$ in biomedical engineering from Texas A\&M University, followed by a postdoctoral fellowship at the University of Illinois at Urbana-Champaign. He has extensive experience in optical coherence tomography and photoacoustic imaging techniques. Currently, he is with PhotoniCare, a medical device company seeking to improve the diagnosis and management of middleear disease.

Ryan G. Porter received his MD degree in 2005, continuing on to complete an internship in general surgery in 2006 and residency in Otolaryngology (H\&NS) in 2010 at Loyola University, Chicago, Illinois. He completed a fellowship at the Michigan Ear Institute in Farmington Hills, Michigan, in 2012. Currently, he is at Carle Foundation Hospital in the Expanding Children's Hearing Opportunities (ECHO) Otolaryngology Clinic.

Michael A. Novak received his BS degree from The Ohio State University, Columbus, Ohio, in 1971, his MD degree from Baylor College of Medicine, Houston, Texas, in 1974, and completed residency in 1979. Currently, he is at Carle Foundation Hospital in the ECHO Otolaryngology Clinic.

Darold R. Spillman Jr. received an associate of arts degree in human resources from the Community College of the Air Force, Maxwell AFB, Georgia, in 2005 and his BA degree in general studies from Eastern Illinois University, Charleston, Illinois, in 2015. He served in the United State Air Force from 1984 to 2007 . He was a 1993 Presidential Point of Light Award nominee and was spotlighted in the Inside Illinois publication.

Eric $\mathrm{J}$. Chaney received his BS degree in biology from the University of Evansville in 1992. From 1993 to 1997, he was a research assistant at the Indiana University School of Medicine, Indiana State University. From 1997 to 2000, he was a transmission electron microscope technician at the University of Illinois at Urbana-Champaign. Since 2000, he has been a research scientist at the Biophotonics Imaging Laboratory, Beckman Institute for Advanced Science and Technology.

Daniel T. McCormick received his BSE degree with distinction in biomedical engineering and electrical engineering from Duke University, Durham, North Carolina, in 1999 and his MS and PhD degrees in electrical and computer engineering from Cornell University, Ithaca, New York, in 2002 and 2003, respectively. He founded Advanced MEMS in 2005 to focus on the development and commercialization of MEMS systems for biophotonic and biomedical applications, with systems employed in numerous human research trials and observational studies.

Stephen A. Boppart received his $\mathrm{PhD}$ in electrical and medical engineering from MIT in 1998, and his MD from Harvard Medical School in 2000. He is an Abel Bliss professor of engineering, a head of the Biophotonics Imaging Laboratory, and a director of the Center for Optical Molecular Imaging. His interests include the development and translation of optical imaging technologies for biological and medical applications. He is a fellow of SPIE, AAAS, IEEE, OSA, BMES, and AIMBE. 